\title{
Enhancement-procedure outcomes in patients implanted with the Precisight multicomponent intraocular lens
}

This article was published in the following Dove Press journal:

Clinical Ophthalmology

\author{
Harvey S Uy ${ }^{1,2}$ \\ Carolina Tesone-Coelho ${ }^{3}$ \\ Harilaos Ginis ${ }^{4}$ \\ 'Peregrine Eye and Laser Institute, \\ Makati, Philippines; ${ }^{2}$ Department of \\ Ophthalmology and Visual Sciences, \\ University of the Philippines, Manila, \\ Philippines; ${ }^{3}$ Department of Research \\ and Development, InfiniteVision \\ Optics, Strasbourg, France; ${ }^{4}$ Research \\ Department, Athens Eye Hospital, \\ Athens, Greece
}

Purpose: Eyes that have undergone phacoemulsification with implantation of a multicomponent intraocular lens (MCIOL) may further undergo an enhancement procedure for correction of residual refractive errors. The enhancement procedure is accomplished by exchanging the front lens used in the primary surgery with another lens containing the correct dioptric power. We evaluated the efficacy and safety of enhancement procedures among eyes that received an MCIOL.

Methods: A total of 25 eyes that had undergone phacoemulsification with implantation of an MCIOL were found to have a residual error of refraction (spherical equivalent $\geq 0.75$ D) 3 months after primary cataract surgery, and underwent further enhancement surgery. The main study outcomes were uncorrected and corrected distance visual acuity, subjective refraction, anterior-chamber depth, pachymetry, and endothelial cell count.

Results: There was a statistically significant improvement in uncorrected distance visual acuity of approximately two lines after enhancement surgery $(0.20 \pm 0.20-0.02 \pm 0.08 \operatorname{logMAR}, P<0.001)$ and a significant decrease in residual spherical equivalent from $1.3 \pm 1.1 \mathrm{D}$ to $0 \pm 0.38 \mathrm{D}(P<0.001)$. There were no statistically significant changes in pre- and postenhancement corrected distance visual acuity, anterior-chamber depth, pachymetry, or keratometry. There was a statistically significant decrease $(2.6 \%)$ in endothelial cell count $(P<0.01)$, which could have been endothelial equilibration from the primary procedure. All enhancement surgeries were uneventful, and no major complications were observed.

Conclusion: The MCIOL-enhancement procedure demonstrates statistical and clinical improvement in uncorrected distance visual acuity and correction of postoperative refractive errors. The Precisight IOL may be a useful choice for patients with high risk of having significant residual refractive errors after primary cataract surgery.

Keywords: residual refractive error, cataract, piggyback lens, multicomponent intraocular lens, intraocular lens exchange, pseudophakic emmetropia

\section{Introduction}

One of the goals of modern cataract surgery is to attain a desired postoperative refraction relevant to the patient's needs. ${ }^{1}$ Despite advances in diagnostic and surgical technology, postoperative refractive surprise and optical intolerance are common causes of patient dissatisfaction, and remain important and challenging issues for ophthalmic surgeons. The number of risk factors related to refractive error after cataract surgery is extensive, ${ }^{2,3}$ increasing the population that might develop poor refractive outcomes. There are several methods for correcting residual refractive errors including corneal refractive surgery, supplementary intraocular lens (IOL), and IOL exchange., ${ }^{4,5}$
Correspondence: Harvey S Uy

Peregrine Eye and Laser Institute, 50 Jupiter Street, Makati, Manila I209,

Philippines

Tel +6328900115

Fax +6325118505

Email harveyuy@yahoo.com
Clinical Ophthalmology 2019:13 107-1/4

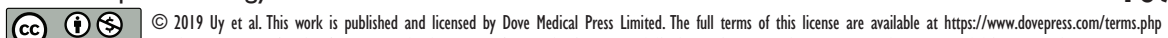
cc) hereby accept the Terms. Non-commercial uses of the work are permitted without any further permission from Dove Medical Press Limited, provided the work is properly attributed. For permision for commercial use of this work, please see paragraphs 4.2 and 5 of our Terms (https://www.dovepress.com/terms.php). 

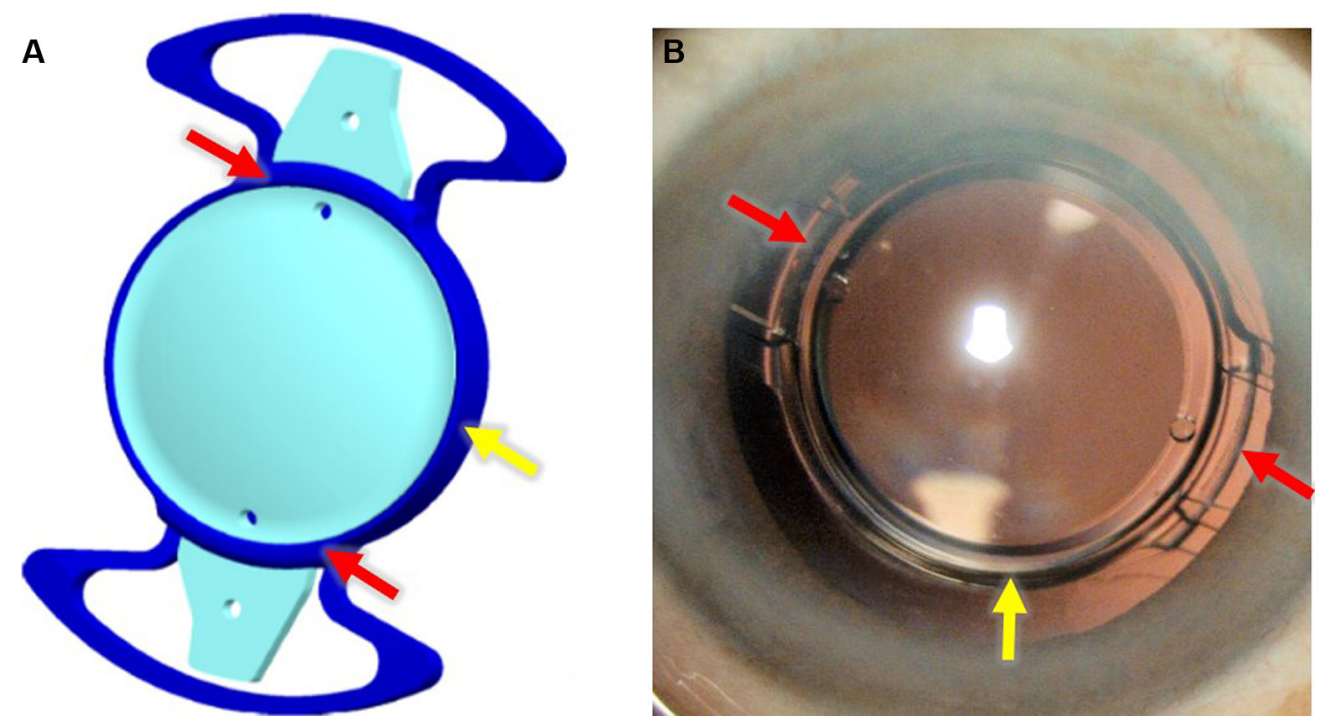

Figure I (A) Illustration of Precisight MCIOL assembly. (B) Implanted Precisight MCIOL at 3 months postenhancement. Note: Red arrows show Precisight MCIOL base-lens bridges; yellow arrows show the base-lens collar. Abbreviation: $\mathrm{MCIOL}$, multicomponent intraocular lens.

Corrective corneal refractive surgery (eg, LASIK) may not be appropriate for all patients, because of preexisting corneal pathology, dry-eye symptoms, ${ }^{6}$ and/or higher-order aberrations that may worsen after corneal surgery and further increase patient dissatisfaction. ${ }^{4,7}$ Supplementary or piggyback IOL implantation is generally easier and less traumatic than IOL exchange, but may be subjected to in interlenticular opacification (ILO), iris chafing with pigment dispersion, and IOL dislocation. Also, significant postoperative rotational instability of supplementary sulcusbased IOLs may lead to suboptimal refractive results. ${ }^{8,9}$

IOL exchange can correct multifocal IOL optical intolerance and even large amounts of ametropia. Unlike laser refractive surgery, IOL exchange also avoids altering the cornea, inducing higher-order aberrations and additional dryeye symptoms. However, the time window for IOL exchange is limited, because resulting capsular fibrosis following the primary cataract procedure may cause adhesion of the IOL to the capsular bag and increases surgical complexity and danger of IOL exchange. ${ }^{2}$ This issue is especially problematic for multifocal IOLs, because it may take 6 months or longer for neuroadaptation to be established. ${ }^{10}$ Earlier IOL exchange ( $<6$ months) may deprive patients of sufficient time for neuroadaptation, while late IOL exchange (more than three months) may lead to significant capsular fibrosis, complicating successful exchange to a monofocal IOL.

A new generation of multicomponent or modular IOLs (MCIOLs) provides a solution to unpredicted refractive errors and multifocal IOL intolerance. ${ }^{11,12}$ The MCIOL is composed of a hydrophobic base lens that serves as a docking station and an exchangeable hydrophilic front lens that is physically connected to the base lens by bilateral "bridge" openings (Figure 1). A minimally invasive surgical exchange (enhancement procedure) of the front-lens component to either a front lens with the correct dioptric power or to a more acceptable spherical/toric optic is performed when indicated. The base lens protects the posterior capsule from damage during the enhancement procedure. We report here the first clinical data regarding the safety and efficacy outcomes of the enhancement procedure to correct residual refractive errors following routine cataract surgery among MCIOL-implanted patients.

\section{Methods}

This prospective, interventional case series involved 25 eyes of 25 patients who underwent phacoemulsification (Phaco) cataract surgery with primary implantation of an MCIOL (Precisight; InfiniteVision Optics, Strasbourg, France) and subsequently underwent refractive enhancement in the form of front-lens exchange for a front-lens optic with new dioptric power. The primary procedures were performed at the Peregrine Eye and Laser Institute, Makati, Philippines between April 2017 and May 2018. The study protocol and informed-consent forms were approved by the Peregrine Eye and Laser Institute Institutional Review Board (PELI-IRB reference: 2015-0023). The tenets of the Declaration for Helsinki were followed in this research. Informed consent was signed by all patients after the nature and possible 
consequences of the study had been explained. All patients received a copy of their signed consent form.

Only one eye of each patient was entered into the study to receive the MCIOL at the time of primary cataract surgery. In patients with bilateral cataracts, the eligible eye for MCIOL surgery was chosen as the eye with worse visual acuity (VA). In cases where preoperative VA was equal, the eye with less corneal astigmatism was selected. For the primary MCIOL implantation, we included eyes with clinically significant cataracts and no other ocular pathology, with maximal expected postoperative corneal astigmatism of $0.75 \mathrm{D}$.

For the enhancement procedure, we included all eyes with expected postenhancement VA of $0.1 \log$ MAR or more, and residual spherical equivalent of at least $0.75 \mathrm{D}$ measured by manifest refraction 3 months after primary implantation with the Precisight MCIOL. Exclusion criteria to undergo the enhancement procedure were unstable capsular bag, ocular diseases that could potentially limit recovery of VA after front-lens exchange, unstable systemic conditions or untreated systemic medical problems, and compromised posterior capsule. The SRK-T (Sanders-Retzlaff-Kraff theoretical) formula was used to calculate the IOL power of the primary MCIOL implant with an assumed A-constant of 118.49 .

The pre-enhancement surgery visit was 3 months after the primary cataract surgery (Phaco). Manifest refraction spherical equivalent (MRSE) and uncorrected and corrected distance VA (UDVA and CDVA, respectively) were determined. For presentation and analysis, all recorded VA data were converted to $\log$ MAR values. Anterior-chamber depth (ACD), pachymetry, and keratometry were assessed using Scheimpflug imaging (Pentacam HD; Oculus Optikgeräte, Wetzlar, Germany). To minimize possible errors and artifacts, pseudophakic ACD was not determined automatically, but using the Pentacam digital caliper to measure the distance from the central corneal endothelium to the IOL anterior surface. Endothelial cell count (ECC) was measured by specular microscopy (CellChek XL; Konan Medical, Irvine, CA, USA).

\section{Enhancement procedure}

The appropriate power for the enhancement front lens was determined using a proprietary calculation formula (https:// www.calculators.infinitevisionoptics.com/Monofocalenhancementcalculator.php). ${ }^{29}$ Residual manifest refraction, pseudophakic ACD, keratometry, and primary front-lens power were used to calculate the best exchange front-lens power to target the plano or least-minus refraction $(0.5 \mathrm{D}$ steps in lens power). All surgical procedures were performed under topical anesthesia by the same surgeon (HSU). An ophthalmic viscosurgical device (OVD) was used to fill the AC using the original Phaco incisions of $2.2 \mathrm{~mm}$. The OVD was injected through one of the two front-lens dialing holes in an effort to lift the front lens away from the base lens without stressing the zonules. The primary front lens was removed from the AC through the original Phaco incision using grasping forceps. A $2.2 \mathrm{~mm}$ injector was used to inject the replacement front lens into the AC. A modified Sinskey hook was then placed through the front-lens tabs or optic hole to place the front-lens haptics into the bridge openings of the base lens (video of surgery is available in Supplementary material). The OVD was irrigated out of the $\mathrm{AC}$ and the space between front and base lenses. Unpreserved prophylactic moxifloxacin was injected into the $\mathrm{AC}$ at the end of the procedure. Postenhancement assessments of VA, biometry, and ECC were performed 2-3 months after enhancement surgery. The protocol to obtain these data was the same as for the pre-enhancement visit. All complications were recorded for all visits.

Data from electronic case-report forms were collected in Microsoft Excel and imported to Statistica 10 (StatSoft, Tulsa, OK, USA) for statistical analysis. There were two outcomes analyzed: performance and safety. The performance of the enhancement was assessed by UDVA and MRSE. The safety outcome looked at CDVA, ECC, ACD, pachymetry, and keratometry. Statistical analysis of parametric variables was performed using Student's $t$-test for dependent samples, while nonparametric data were analyzed using Wilcoxon matched-pair tests. In all cases, statistical significance was set to $\alpha<0.05$, adjusted with Bonferroni correction for each group of hypotheses.

\section{Results}

The study evaluated 25 eyes from 25 patients that underwent enhancement procedures at least 3 months after previous uneventful primary Precisight implantation: 19 (76\%) were female and six (24\%) were male. The mean age was $66 \pm 8.28$ (52-81) years. The mean axial length was $23.3 \pm 0.5$ (22.1-24.3) $\mathrm{mm}$. There were no fibrosis around the frontlens tabs, no stickiness between the lenses, and no difficulty in exchanging the front lens in any eyes. All enhancement surgeries were performed without complications.

Figure 2 shows the descriptive statistics of UDVA and CDVA in logMAR before and after enhancement surgery. A significant difference was detected for $\operatorname{UDVA}(P<0.001)$. Patients gained an average of two lines after the enhancement 


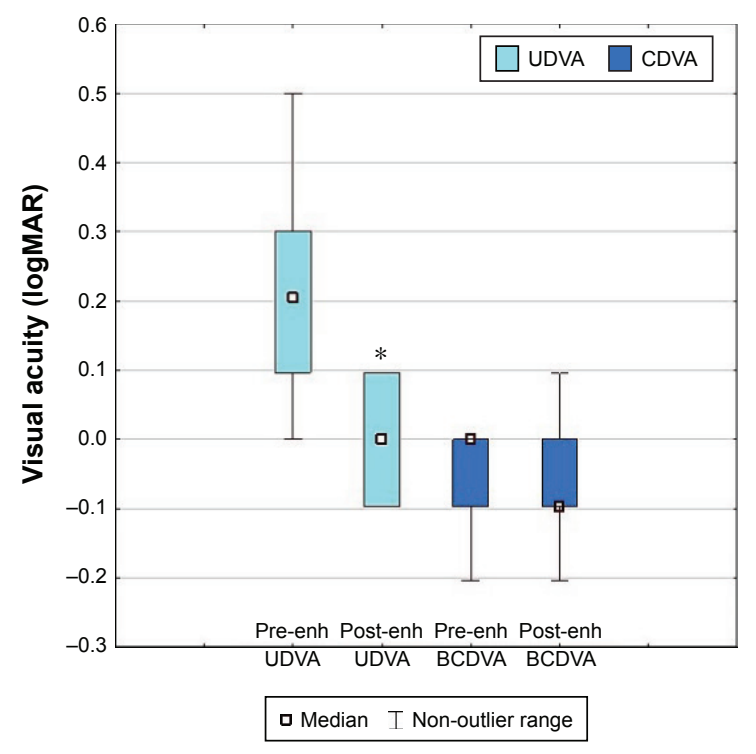

Figure 2 UDVA and CDVA before and after the enhancement procedure. Notes: $* P<0.05$ vs respective pre-enhancement (Pre-enh) parameter (Wilcoxon matched-pair tests). Colored boxes are $25 \%-75 \%$.

Abbreviations: CDVA, corrected distance visual acuity; Post-enh, postenhancement; UDVA, uncorrected DVA.

procedure $(0.20 \pm 0.20 \log$ MAR before vs $0.02 \pm 0.08 \log$ MAR after). Postenhancement CDVA remained unchanged $(-0.10 \pm 0.10 \log$ MAR before vs $-0.06 \pm 0.07 \log$ MAR after, $P=0.25)$. Two eyes lost one line of CDVA ( $0-0.1$ and -0.1 to $0 \log$ MAR). For those patients, postenhancement UDVA was equal to postenhancement CDVA, showing that their best-possible vision had been achieved. All eyes were stable, with a CDVA of at least $0.1 \log$ MAR.

Figure 3 shows the distribution of MRSE before and 3 months after enhancement surgery. Paired $t$-tests revealed a significant reduction in MRSE after enhancement $(P<0.001)$. Prior to enhancement, MRSE was $1.3 \pm 1.1(-1.6$ to 3.0) D, and after enhancement MRSE had decreased to $0 \pm 0.38$ (-0.6 to 1.0$)$ D.

Biometry and ECC data before and after enhancement are shown in Table 1. ACD, pachymetry, and corneal astigmatism were unchanged after enhancement. There was a small but significant statistical reduction in ECC (less 2.6\%) after enhancement when compared to the pre-enhancement value $(P<0.01)$.

No intraoperative complications developed during or after enhancement procedures. For the time period analyzed here, no postoperative major complications related to implantation of a multicomponent IOL were observed (eg, no pupillary block). At 3 months postenhancement, one eye (4\%) had moderate iritis that was treated with topical corticosteroid drops, and three eyes (12\%) developed mild, clinically insignificant pigment dispersion. One patient had increased intraocular pressure, which was controlled with pressure-lowering drops. All these sequelae resolved within 3 months. There were no cases of significant posterior capsular opacification that resulted in a need for YAG laser capsulotomy up to 1 year after enhancement. No ILO was observed.

\section{Discussion}

Cataract surgery is now considered a refractive procedure, with many patients expecting good visual and refractive outcomes without the need for glasses. Therefore, an unsatisfactory visual result due to residual refractive errors creates an undesirable and frustrating situation for both patients and physicians. Emmetropia (spherical equivalent -0.5 to $0.5 \mathrm{D}$, associated with $<1 \mathrm{D}$ astigmatism) is the goal of most cataract surgeons. ${ }^{4}$ However, this result is consistently achieved in only $73.5 \%$ of eyes planned for emmetropia. ${ }^{3}$ In some national registries, the proportion of emmetropic patients can be as low as $55 \% .{ }^{1}$ Certain factors (eg, previous corneal refractive surgery, concomitant eye diseases) are strongly correlated with postoperative ametropia. ${ }^{2,4}$ Despite advances in biometry and IOL technology, it was recently shown that the number of risk factors for postoperative refractive surprise was larger than had been previously reported (eg, poor preoperative VA, corneal opacity, and surgical complications). ${ }^{2}$

The correction of pseudophakic ametropia can be achieved by total primary lens explantation and replacement, LASIK, or implantation of a secondary sulcus piggyback lens. ${ }^{4,7}$ This paper demonstrates that the Precisight MCIOL may be a good choice of implant for patients with higher risks of postoperative refractive surprises, thanks to its adjustable optics.

We demonstrated here that the enhancement procedure of the Precisight is effective and safe to correct residual refractive errors after primary IOL implantation. After enhancement, patients had gained on average two lines of uncorrected distance vision, indicating a substantial improvement in refractive outcomes. Other studies have also shown significant improvement and accurate prediction of refractive outcomes after secondary piggyback implantation. ${ }^{13-18}$ In both cases, these results are probably due to the predictable positioning of the secondary lens and the optimized formulae used to calculate enhancement power.

After the exchange of the Precisight front lens, $64 \%$ of patients achieved $20 / 20$ or better and $100 \%$ achieved at least 20/32 UDVA. The improvement in UDVA was not only statistically but also clinically significant. Similar results were found in a recent study that used the sulcus AddOn IOL 
A

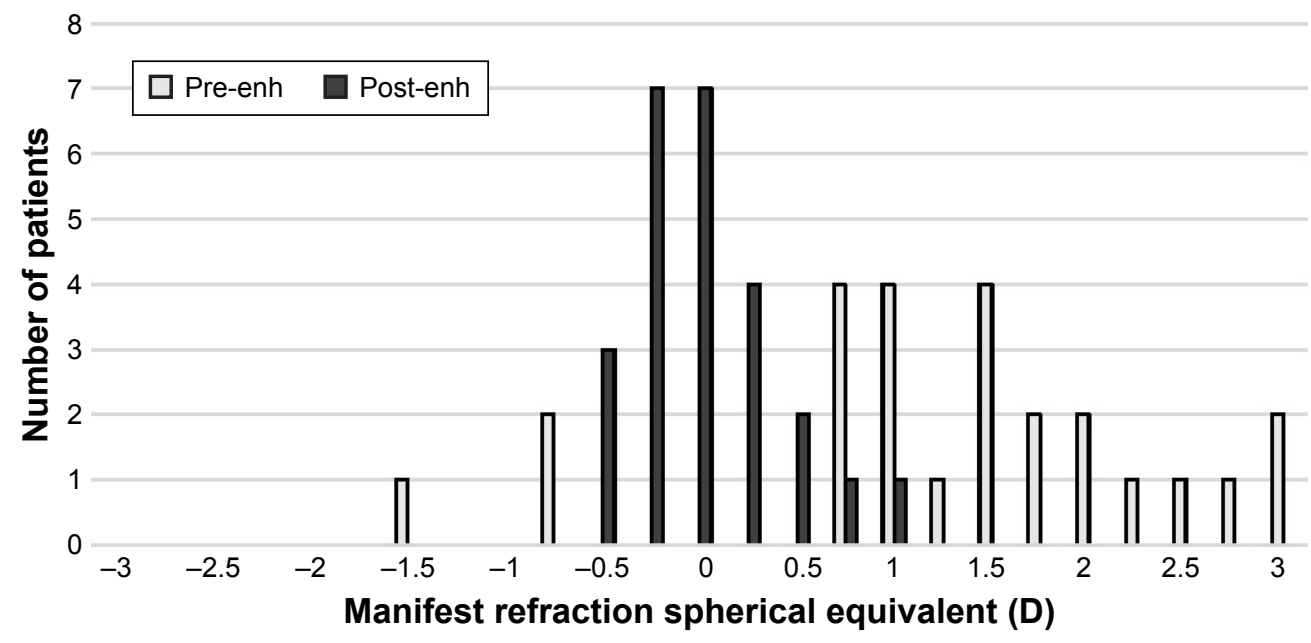

B

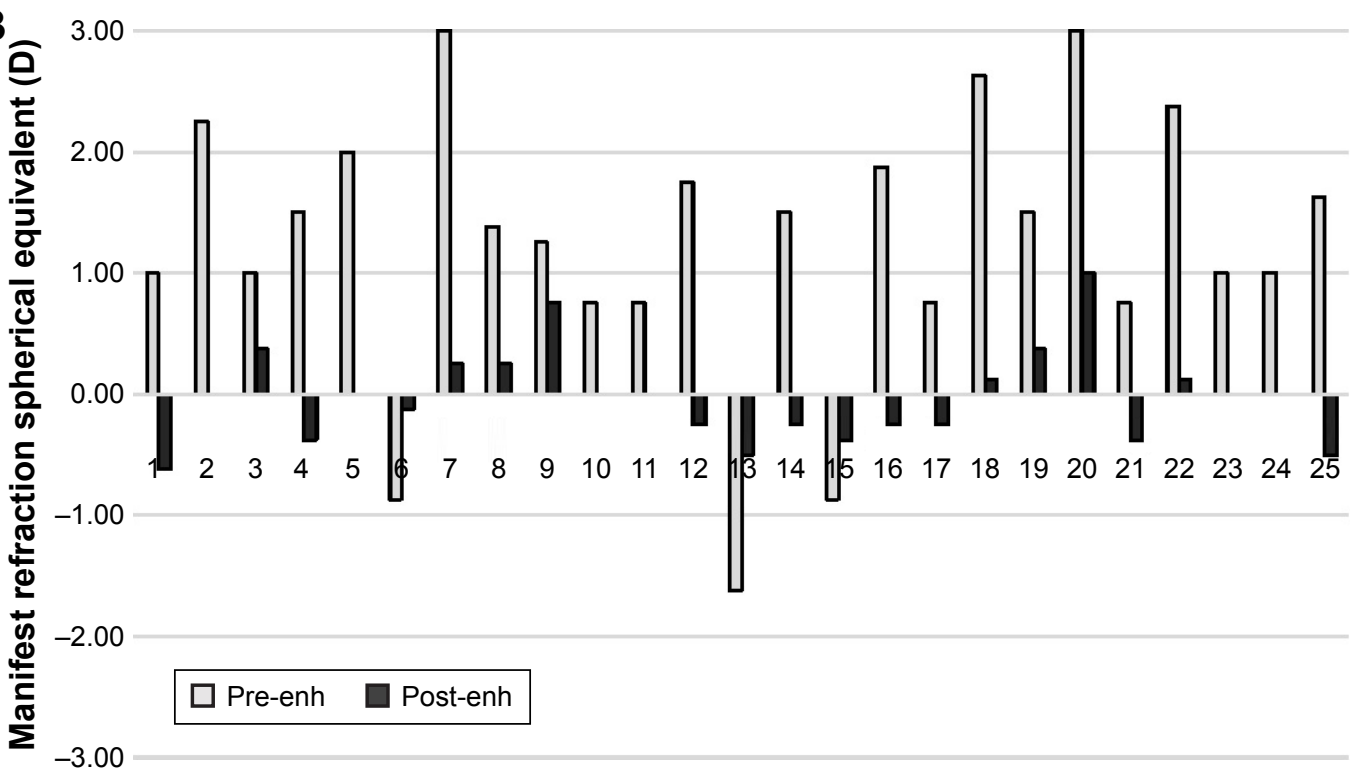

Figure 3 (A) Distribution of the patients and (B) individual manifest refraction spherical equivalent before and 3 months after the enhancement (enh) procedure. Note: Lack of black bar in $\mathbf{B}$ means that the manifest refraction spherical equivalent was equal to zero.

(1stQ, Mannheim, Germany). ${ }^{16}$ In terms of residual refraction, our study showed that $100 \%$ of eyes were within $\pm 1 \mathrm{D}$ : $88 \%$ of patients were within the emmetropia range -0.5 to $0.5 \mathrm{D}$, with $60 \%$ within $\pm 0.25 \mathrm{D}$. Interestingly, the results are similar to those found with the AddOn IOL, in which 57\% and
$76 \%$ of eyes were within $\pm 0.25 \mathrm{D}$ and $\pm 0.5 \mathrm{D}$, respectively. ${ }^{16}$ It is noteworthy to mention that refractive results after more recent studies of piggyback IOL implantation have improved, with results showing $93 \%-100 \%$ of patients within $\pm 0.5 \mathrm{D}$ of target refraction. ${ }^{13,17,18}$ Laser refractive surgery also

Table I Comparison of the biometry and ECC at 3 months after cataract surgery (pre-enhancement) and 3 months after enhancement procedure (postenhancement)

\begin{tabular}{l|l|l|l}
\hline & Pre-enhancement & Postenhancement & P-value \\
\hline ACD $($ endothelium to anterior-lens surface, $\mathrm{mm})$ & $3.8 \pm 0.40(3.1-4.4)$ & $3.8 \pm 0.48(3.1-4.8)$ & 0.14 \\
\hline Pachymetry $(\mu \mathrm{m})$ & $540 \pm 28(484-612)$ & $540 \pm 25(492-603)$ & 0.94 \\
\hline $\mathrm{CA}\left(\mathrm{K}_{1}-\mathrm{K}_{2}\right), \mathrm{D}$ & $-0.7 \pm 0.3(-1.2$ to -0.3$)$ & $-0.7 \pm 0.3(-1.2$ to -0.1$)$ & 0.50 \\
\hline ECC $\left(\right.$ cells $\left./ \mathrm{mm}^{2}\right)$ & $2,845 \pm 226(2,364-3,257)$ & $2,769 \pm 274(2,247-3,215)$ & 0.009 \\
\hline
\end{tabular}

Notes: Data presented as mean \pm SD (range). $P$-values refer to paired $t$-tests.

Abbreviations: $A C D$, anterior-chamber depth; CA, corneal astigmatism; ECC, endothelial cell count. 
provides very good refractive results and is considered more accurate than lens-based procedures. ${ }^{4}, 15$ The results found for pseudophakic patients treated with LASIK are similar to Precisight results, with $92.3 \%$ of eyes achieving a final spherical equivalent within $\pm 0.5 \mathrm{D}$ from emmetropia and $100 \%$ within \pm 1.0 D. $^{15}$

Three patients had a residual refraction between \pm 0.5 and $\pm 1.0 \mathrm{D}$. The spherical equivalent of those three patients were $-0.63,0.75$, and $1 \mathrm{D}$ (Figure 3B; patients 1, 9, and 20, respectively). The first was deliberately targeted for slight myopia, as the plano target-exchange front lens was not available, which could explain this small deviation from the $\pm 0.5 \mathrm{D}$ standard of emmetropia. For the other two patients, despite targeting for plano, residual postenhancement refractive error between \pm 0.5 and $\pm 1.0 \mathrm{D}$ was observed. The $\mathrm{ACD}$ was highly stable for both patients, and thus a possible shift in IOL position cannot explain this second refractive surprise. It is important to stress that small errors in calculating the new lens power may have occurred because of the early evolution of the technology and the need to refine the IOL A-constant. Still, the exchange front-lens calculation and the IOL itself have had a promising beginning, since emmetropia was achieved in almost $90 \%$ of patients and no patients had refractive errors $> \pm 1 \mathrm{D}$.

The vast majority of patients experienced stability of improvement in CDVA after enhancement. Only two eyes experienced loss of just one line of CDVA. None of the eyes lost more than one line of CDVA. We attribute this decrease to measurement "noise" or patient-related factors, such as fatigue and dry eye. Both of these patients were happy with their outcomes: one patient's CDVA dropped from 20/16 to $20 / 20$, and the other from $20 / 20$ to $20 / 25$.

In regard to safety outcomes, the present study showed no major complications with the Precisight exchange procedure. Mild, transient pigment dispersion was observed in three eyes (12\%) and resolved after 2 weeks to 3 months with topical corticosteroid therapy, and was likely related to mild iris trauma during the implantation procedure itself. In-the-bag placement of the MCIOL prevents IOL-posterior iris contact and pigment dispersion. Over a 3-month observation period, none of the eyes developed pigment dispersion or pigmentary glaucoma after cessation of corticosteroid drops. Schrecker et a $1^{19}$ observed the same rate of mild pigment dispersion after piggyback IOL implantation with no clinical consequences. Mild pigment dispersion has been observed in other studies using modern piggyback IOLs. The percentage of pigment dispersion varied: $8 \%-27 \% .^{20,21}$

ILO is a concern with piggyback implantation. Placing a piggyback lens in the sulcus may prevent ILO and its related hyperopic shift. When sulcus positioning is not possible, a generous capsulorhexis that is larger than the optics should be performed to allow the posterior and anterior capsules to fuse and thereby sequester the proliferative epithelial cells. ${ }^{7}$ We observed here that no interlenticular migration of cells occurred using the Precisight MCIOL. This complication is avoided by creating a capsulorhexis larger than the optic at the time of the Phaco. ${ }^{9}$ Most importantly, however, is a "collar" surrounding the base-lens optic edge (Figure 1), which prevents cells from migrating into the interlenticular space. Implantation of the Precisight MCIOL in the capsular bag does not interfere with the enhancement procedure, because no fibrosis was observed capturing in the front-lens haptics.

There was a small but statistically significant $(P<0.01)$ reduction in the ECC $(2.6 \%)$ when compared to the preenhancement time point. It is well known that the paracentral and peripheral regions of the corneal endothelium decline with age at a rate of $0.2 \%-0.6 \%$ per year. ${ }^{22,23}$ Moreover, the decline observed in this study is comparable to other studies that reported EC reductions of $5 \%-13.6 \%$ at the 6 -month postoperative (primary cataract surgery) period. As such, the cell loss noted after enhancement surgery may still reflect the endothelial equilibration from the primary surgery and not any additional loss from the enhancement. ${ }^{24-27}$ The implementation of newer surgical technologies, such as torsional ultrasound and viscoelastic devices, and aspects of patients' preexisting medical history may lead to varying degrees of ECC loss after cataract surgery. ${ }^{28}$ The follow-up presented here ( 3 months postenhancement) is on average 6 months after Phaco, the period in which most, but not all, changes in ECC are usually observed. ${ }^{27}$ Further analysis is needed to correlate the decline in number of cells with the enhancement procedure or Phaco. Still, this decrease of $2.6 \%$ is much lower than the expected ECC loss after cataract surgery.

In regard to surgical ease and safety, the Precisight MCIOL is injected preassembled for the primary cataract procedure, just like a standard IOL. During the enhancement procedure, the Precisight MCIOL system allows for a minimally invasive exchange of the front-lens component of the optical system. Since the posterior capsular bag is protected by the base-lens optic, the risk of capsular tear and vitreous loss is greatly reduced. Because both lenses are coupled and the base component has become fixed with capsular fibrosis in its original position, the chance of postenhancement IOL rotation is minimized. Therefore, the MCIOL system provides a safe, easily performed alternative solution for cases that would otherwise require a much more difficult full lens exchange. Finally, because the posterior capsular bag is protected by the base-lens optic, the exchange of the 
front lens can theoretically be performed even after Nd:YAG laser capsulotomy.

\section{Limitations}

There are some limitations in the present study: a small sample, which limited the analysis to a univariate association, and a limited follow-up. A future prospective study with longer follow up and more patients is necessary to confirm long-term results and eventually account for potentially important confounders of the enhancement procedure of the Precisight MCIOL.

\section{Conclusion}

The present study demonstrates that the Precisight MCIOL may be a good choice for patients with a high risk of residual refractive error or optical intolerance (multifocal optics) after cataract surgery. The enhancement procedure is associated with a significant improvement in refractive and visual outcomes after cataract surgery. The enhancement procedure has not demonstrated any major surgical or clinical complications. Inthe-bag placement of the implant does not impair the enhancement procedure. This technology avoids the corneal side effects of laser enhancements and the surgical complications related to full-lens explantation or traditional piggyback lenses.

\section{Acknowledgment}

We thank Dr Corinne Wittmer for writing assistance and scientific advice.

\section{Author contributions}

HSU contributed to data acquisition, interpretation, writing, and critically revised the paper. CTC contributed to conception, design, data analysis, drafting, and manuscript preparation. HG contributed to data acquisition and interpretation and critically revised the paper. All authors contributed toward data analysis, drafting and critically revising the paper, gave final approval of the version to be published and agree to be accountable for all aspects of the work in ensuring that questions related to the accuracy and integrity of work are investigated and resolved.

\section{Disclosure}

HSU is an investigator for InfiniteVision Optics. CTC is an InfiniteVision Optics employee. HG is a consultant for InfiniteVision Optics.

\section{References}

1. Behndig A, Montan P, Stenevi U, Kugelberg M, Zetterström C, Lundström M. Aiming for emmetropia after cataract surgery: Swedish National Cataract Register study. J Cataract Refract Surg. 2012;38(7):1181-1186.
2. Lundström M, Dickman M, Henry Y, et al. Risk factors for refractive error after cataract surgery: Analysis of 282811 cataract extractions reported to the European Registry of Quality Outcomes for cataract and refractive surgery. J Cataract Refract Surg. 2018;44(4):447-452.

3. Lundstrom MB, Dickman L, Henry M, et al. ESCRS - EUROQUOAnnual Report 2016. European Society of Carataract and Refractive Surgery; 2016.

4. Alio JL, Abdelghany AA, Fernández-Buenaga R. Management of residual refractive error after cataract surgery. Curr Opin Ophthalmol. 2014;25(4):291-297.

5. Leysen I, Bartholomeeusen E, Coeckelbergh T, Tassignon MJ. Surgical outcomes of intraocular lens exchange: five-year study. $J$ Cataract Refract Surg. 2009;35(6):1013-1018.

6. Raoof D, Pineda R. Dry eye after laser in-situ keratomileusis. Semin Ophthalmol. 2014;29(5-6):358-362.

7. Sáles CS, Manche EE. Managing residual refractive error after cataract surgery. J Cataract Refract Surg. 2015;41(6):1289-1299.

8. Meyer JJ, Mcghee CN. Supplementary, sulcus-fixated intraocular lens in the treatment of spherical and astigmatic refractive errors in pseudophakic eyes after keratoplasty. Cornea. 2015;34(9):1052-1056.

9. Trivedi RH, Werner L, Apple DJ, Pandey SK, Izak AM. Post cataract-intraocular lens (IOL) surgery opacification. Eye (Lond). 2002;16(3):217-241.

10. Rosa AM, Miranda ÂC, Patrício MM, et al. Functional magnetic resonance imaging to assess neuroadaptation to multifocal intraocular lenses. J Cataract Refract Surg. 2017;43(10):1287-1296.

11. Portaliou DM, Grentzelos MA, Pallikaris IG. Multicomponent intraocular lens implantation: two-year follow-up. J Cataract Refract Surg. 2013;39(4):578-584.

12. Portaliou DM, Kymionis GD, Pallikaris IG. Multi-component adjustable intraocular lenses: a new concept in pediatric cataract surgery. J Refract Surg. 2014;30(1):62-66.

13. Basarir B, Kaya V, Altan C, Karakus S, Pinarci EY, Demirok A. The use of a supplemental sulcus fixated IOL (HumanOptics Add-On IOL) to correct pseudophakic refractive errors. Eur J Ophthalmol. 2012;22(6): 898-903.

14. Falzon K, Stewart OG. Correction of undesirable pseudophakic refractive error with the Sulcoflex intraocular lens. J Refract Surg. 2012;28(9): 614-619.

15. Fernández-Buenaga R, Alió JL, Pérez Ardoy AL, et al. Resolving refractive error after cataract surgery: IOL exchange, piggyback lens, or LASIK. J Refract Surg. 2013;29(10):676-683.

16. Gundersen KG, Potvin R. A review of results after implantation of a secondary intraocular lens to correct residual refractive error after cataract surgery. Clin Ophthalmol. 2017;11:1791-1796.

17. Hassan AHA, Sayed KM, Elagooz M, Elhawary AM. Refractive Results: Safety and Efficacy of Secondary Piggyback Sensar AR40 Intraocular Lens Implantation to Correct Pseudophakic Refractive Error. J Ophthalmol. 2016;2016(1):1-5.

18. Kahraman G, Amon M. New supplementary intraocular lens for refractive enhancement in pseudophakic patients. J Cataract Refract Surg. 2010;36(7):1090-1094.

19. Schrecker J, Blass S, Langenbucher A. Silicone-diffractive versus acrylic-refractive supplementary iols: visual performance and manual handling. J Refract Surg. 2014;30(1):41-48.

20. Liekfeld A, Ehmer A, Schröter U. Visual function and reading speed after bilateral implantation of 2 types of diffractive multifocal intraocular lenses: Add-on versus capsular bag design. $J$ Cataract Refract Surg. 2015;41(10):2107-2114.

21. Schrecker J, Langenbucher A. Visual Performance in the Long Term With Secondary Add-on Versus Primary Capsular Bag Multifocal Intraocular Lenses. $J$ Refract Surg. 2016;32(11):742-747.

22. Grossniklaus HE, Nickerson JM, Edelhauser HF, Bergman LA, Berglin L. Anatomic alterations in aging and age-related diseases of the eye. Invest Ophthalmol Vis Sci. 2013;54(14):ORSF23-ORSF27.

23. Güell JL, El Husseiny MA, Manero F, Gris O, Elies D. Historical Review and Update of Surgical Treatment for Corneal Endothelial Diseases. Ophthalmol Ther. 2014;3(1-2):1-15. 
24. Dick HB, Kohnen T, Jacobi FK, Jacobi KW. Long-term endothelial cell loss following phacoemulsification through a temporal clear corneal incision. J Cataract Refract Surg. 1996;22(1):63-71.

25. Faramarzi A, Javadi MA, Karimian F, et al. Corneal endothelial cell loss during phacoemulsification: bevel-up versus bevel-down phaco tip. J Cataract Refract Surg. 2011;37(11):1971-1976.

26. Nayak BK, Shukla RO. Effect on corneal endothelial cell loss during phacoemulsification: fortified balanced salt solution versus Ringer lactate. J Cataract Refract Surg. 2012;38(9):1552-1558.
27. Werblin TP. Long-term endothelial cell loss following phacoemulsification: model for evaluating endothelial damage after intraocular surgery. Refract Corneal Surg. 1993;9(1):29-35.

28. Rosado-Adames N, Afshari NA. The changing fate of the corneal endothelium in cataract surgery. Curr Opin Ophthalmol. 2012;23(1):3-6.

29. InfiniteVision Optics Monofocal Enhancement calculator [webpage on the internet]. Strasbourg: InfiniteVision Optics 2018 [Updated July 2017]. Available from: https:/www.calculators.infinitevisionoptics.com/ Monofocalenhancementcalculator.php. Accessed December 24, 2018.
Clinical Ophthalmology

\section{Publish your work in this journal}

Clinical Ophthalmology is an international, peer-reviewed journal covering all subspecialties within ophthalmology. Key topics include: Optometry; Visual science; Pharmacology and drug therapy in eye diseases; Basic Sciences; Primary and Secondary eye care; Patient Safety and Quality of Care Improvements. This journal is indexed on

\section{Dovepress}

PubMed Central and CAS, and is the official journal of The Society of Clinical Ophthalmology (SCO). The manuscript management system is completely online and includes a very quick and fair peer-review system, which is all easy to use. Visit http://www.dovepress.com/ testimonials.php to read real quotes from published authors. 